\title{
Thermal Diffusion of Isotopes in Pure Liquids
}

\author{
A. LoDDing \\ Physics Department, Chalmers University of Technology, Gothenburg, Sweden \\ (Z. Naturforschg. 21 a, 1348-1351 [1966] ; received 18 March 1966)

\begin{abstract}
A theoretical treatment is given of the recently discovered thermotransport effect in pure liquid metals. The isotope effect is related to the diffusive mass flow by a proportionality factor familiar from electrotransport. The mass flow is given primarily by the temperature dependence of the mean size or amount of density fluctuations in the liquid. Very little activation energy is required for diffusive motion, which takes place by cooperative position adjustments of neighbor atoms. The mean displacement length of the diffusing cluster is by an order of magnitude smaller than the effective cluster diameter. The liquid model suggested is coherent with models based on evidence from other experimental methods.
\end{abstract}

It was discovered two years ago ${ }^{1}$, that a considerable de-mixing of isotopes takes place in pure liquid lithium metal, when a temperature gradient is imposed. The same effect was since found in other molten metals ${ }^{2}$. In all cases the light isotope was enriched at the hot end. The maximal steady-state separation factor (i. e. the abundance ratio of the isotopes at the cold end of the cell, divided by the abundance ratio at the hot end) was for lithium (masses 6 and 7 ) about 1.04, corresponding to the temperature interval $350^{\circ}-525^{\circ} \mathrm{C}$. In gallium (masses 69 and 71 ) the temperature interval $50^{\circ}$ to $500{ }^{\circ} \mathrm{C}$ gave a separation factor of about 1.03 . The same order of magnitude was obtained in the other liquid metals investigated.

As thermal diffusion (SôRET-effect) of isotopes represents the purest possible example of thermotransport, it should be possible to draw significant theoretical conclusions.

On account of numerous evidence of close-range order in liquids and on the reasonable assumption that at any moment the percentage of particles engaged in diffusive position adjustment relative to its neighbors is small, the concept can be used of a pseudo-lattice, consisting of the bulk of momentarily ordered atoms. This, rather than the container, will serve as a reference system for atom transport motion in a liquid. With such a model, a certain analogy with a solid-state approach appears justified.

1 A. Ott and A. LundÉn, Z. Naturforschg. 19a, 822 [1964].

2 A. Lodding and A. Otт, Z. Naturforschg. 21 a, 1344 [1966].

3 See e. g., P G. Shewmon, Diffusion in Solids, McGraw-Hill, New York 1963.

${ }^{4}$ K. Furukawa, Nature 184, 1209 [1959].

5 K. Furukawa, Sci. Rep. Res. Inst. Tohoku Univ., Ser. A, 12, 368 [1960].
To begin with, as in solid-state diffusion ${ }^{3}$ it can be assumed that, in order to make a displacement, a particle needs an energy $E_{\mathrm{m}}$ ("activation energy for motion") to overcome a potential barrier. If the diffusion takes place by defects, e. g. vacancies, an "activation" energy $E_{\mathrm{v}}$ for defect formation is also required. Vacancies in liquids have a different meaning from those in solids, but the melt does have a great concentration of voids of various sizes $^{4,5}$, and generally the nearby presence of a density fluctuation will be conducive to a diffusive step. Only if the main transport mechanism is some kind of a rotation, the formation energy will be zero. The notion of activation energies in liquids has been under discussion ${ }^{6-8}$. Although the temperature behavior of diffusion coefficients has shown certain deviations from the Arrhenius type relationship, within not too wide temperature intervals straight lines are obtained. In the present treatment, a Boltzmann factor dependence of displacement probabilities will be used (at worst a useful approximation). It will not be assumed, however, that diffusion necessarily takes place by single atoms making jumps of a definite length. To allow for the possibility of cooperative motion, suggested by several authors ${ }^{6,8,9}$, the diffusing particles will be thought of as clusters of several atoms, and the displacement lengths as ranging from zero to several interatomic distances. It is, in fact, the purpose of

${ }^{6}$ R. A. Swalin, Acta Met. 7, 336 [1959].

7 H. A. Walls and W. R. Upthegrove, Acta Met. 12, 461 [1964].

8 M. H. Cohen and D. Turnbull, J. Chem. Phys. 31, 1164 [1959].

9 A. Otr and A. Lodding, Z. Naturforschg. 20 a, 1578 [1965]. 
this treatment to show that experiments indicate that the mean displacement length is considerably less than the mean distance between nearest neighbor particle centers.

\section{a) Isothermal Diffusion}

Let the binary liquid isotope mixture consist of components 1 and 2 . With the pseudo-lattice model in mind, denoting by $\bar{l}$ the positive root mean square displacement, one can derive the diffusion coefficient $D_{i}$ of the component $i(i=1$ or 2$)$ by taking the difference of the forward and backward fluxes between two planes a distance $\bar{l}$ apart (cf. ref. $\left.{ }^{3}\right)$ :

$j_{i}^{+}=-\left(n_{i}\right) \gamma v_{i} \bar{l} \exp \left[-\left(E_{\mathrm{m}}+E_{\mathrm{v}}\right) / R T\right]$,

$j_{i}{ }^{-}=-\left(n_{i}+\bar{l} \nabla n_{i}\right) \gamma \nu_{i} \bar{l} \exp \left[-\left(E_{\mathrm{m}}+E_{\mathrm{v}}\right) / R T\right]$

Here $\gamma$ is a term containing i. a. a geometrical constant and an entropy term; $v_{i}$ is a (mass dependent) frequency term. The energy $E_{\mathrm{m}}$ denotes, as discussed above, the "activation" energy necessary for one atom to overcome a barrier, whereas $E_{\mathrm{v}}$ decides the likelihood of a suitable void (or other defect necessary for motion) being in a nearby position. Both $E_{\mathrm{m}}$ and $E_{\mathrm{v}}$ are counted per mole of atomic species. If motion takes place by clusters, $E_{\mathrm{m}}$ thus denotes the necessary energy at the particle site, but $E_{\mathrm{v}}$ represents an energy pertaining to a configuration at the "outskirt" of the cluster. With this point in mind, one can nevertheless see that under isothermal conditions the Eqs. ( $1 \mathrm{a})$ and $(1 \mathrm{~b})$ are valid quite independent of whether single atoms or clusters are the diffusing species.

The flux difference is

$$
j_{i}=j_{i}^{+}-j_{i}^{-}=-\left(\nabla n_{i}\right) \gamma v_{i} \bar{l} \exp \left[-\left(E_{\mathrm{m}}+E_{\mathrm{v}}\right) / R T\right]
$$

and the diffusion coefficient follows from comparison with Fick's lst law, such that

$$
D_{i}=f \gamma \nu_{i} \bar{l}^{2} \exp \left[-\left(E_{\mathrm{m}}+E_{\mathrm{v}}\right) / R T\right],
$$

where also the BARDEEN-HERRING correlation factor $f$ has been taken into account. It can be shown ${ }^{9}$ that

10 A. Klemm, J. Chim. Phys. 60, 1-2, 237 [1963] ; also new results quoted in personal communication.

11 A. Klemm, E. Lindholm, and A. LundÉn, Z. Naturforschg. 7 a, 560 [1952]. the experimental inter-diffusion coefficient of isotopes in a binary liquid mixture is given by

$$
D=c_{1} D_{2}+c_{2} D_{1},
$$

where $c_{1,2}$ are the relative particle concentrations.

From the measurements of the temperature dependence of $D$, the self-diffusion activation energy $E_{\mathrm{m}}+E_{\mathrm{v}}$ can evidently be obtained.

The dependence of $D_{i}$ on isotope mass $M_{i}$ may be estimated by comparison with experimental isotope mass dependence of ionic mobilities $u_{i}$ in molten salts ${ }^{10,11}$, via the equation

$$
\begin{gathered}
D_{1} / D_{2} \cong u_{1} / u_{2}=\left(M_{1} / M_{2}\right)^{a}, \\
\partial \ln D_{i} / \partial \ln M_{i} \cong a .
\end{gathered}
$$

It has been found ${ }^{\mathbf{1 0}}$ that the isotope factor $a$ (which would be $-1 / 2$ if single atoms could be considered as jumping from one rigid cage to another) is in liquid salts satisfactorily represented by $a \cong-0.1$. The qualitative justification of using this assessment also to liquid metals has been shown when explaining the phenomenon of isotope electromigration in metals ("HAEFFNER-effect") ${ }^{12,13}$.

\section{b) Thermal Diffusion}

Neglecting the temperature dependence of density and of $v_{i}$, one can express the velocity (relative to the pseudo-lattice) of an atom of isotope $i$ (or of a cluster containing it), in a temperature gradient, as the difference of the forward and the backward velocity, analogously with Eqs. (1) (using $j_{i}=n_{i} v_{i}$ ), such that

$$
\begin{aligned}
v_{i}^{+}= & \gamma v_{i} \bar{l} \exp \left[-E_{\mathrm{m}} / R T\right] \\
& \cdot \exp \left[-E_{\mathrm{v}} / R T(1+\varphi \bar{l} \cdot \nabla \ln T)\right], \\
v_{i}^{-}= & \gamma v_{i} \bar{l} \exp \left[-E_{\mathrm{m}} / R T(1+\varphi \bar{l} \cdot \nabla \ln T)\right] \\
& \cdot \exp \left[-E_{\mathrm{v}} / R T\right] .
\end{aligned}
$$

Hence, using also Eq. (1) for $i=1,2$ and assuming the same transport mechanism for self-diffusion and thermal diffusion,

$v_{1}-v_{2}=-\frac{\varphi}{f} \cdot\left(D_{1}-D_{2}\right) \cdot \nabla \ln T \frac{E_{\mathrm{m}}-E_{\mathrm{v}}}{R T}$.

The energy difference $E_{\mathrm{m}}-E_{\mathrm{v}}$ is usually called

12 A. Lodding, in Encyclopedia of Electrochemistry, Reinhold, New York 1964, p. 491; also J. Phys. Chem. Solids, in press.

13 A. KLемm, Z. Naturforschg. 9 a, 1031 [1954]. 
"heat of transport" 14 . The factor $\varphi$, important in the present reasoning, gives the distance $\varphi \bar{l}$ between the center of the cluster and the center of the particular void which makes cluster displacement by a distance $\bar{l}$ possible.

As long as $\Delta M=M_{1}-M_{2} \ll M$, one can put

$$
\left(v_{1}-v_{2}\right) /\left(M_{1}-M_{2}\right) \cong \partial v / \partial M .
$$

From Eq. (5 a) it is obvious that $\partial \ln v=\partial \ln D$, and, using also Eq. ( $5 \mathrm{~b}$ )

$$
v_{1}-v_{2}=-\frac{a D}{f} \frac{\Delta M}{M} \frac{\varphi\left(E_{\mathrm{m}}-E_{\mathrm{v}}\right)}{R T} \nabla \ln T .
$$

\section{c) Interpretation of Experiments}

The entity obtained in thermal diffusion measurements is the steady-state separation factor

$$
Q_{\max }=\frac{\left(c_{2} / c_{1}\right)_{\mathrm{C}}}{\left(c_{2} / c_{1}\right)_{\mathrm{H}}},
$$

where the suffixes $\mathrm{C}$ and $\mathrm{H}$ refer to the "cold end", respectively the "hot end" of the cell.

In the steady state,

$$
v_{1} c_{1}=D_{\text {eff }}\left(\partial c_{1} / \partial x\right)=-v_{2} c_{2},
$$

where $x$ is the length coordinate of a cylindrical capillary cell and $D_{\text {eff }}$ is the effective self-diffusion coefficient, equal to $\left(D+D^{\prime}\right), D^{\prime}$ denoting the convective term. Eqs. (9) and (10) lead to

$$
v_{2}-v_{1}=D_{\text {eff }}(\partial \ln Q / \partial x) \text {. }
$$

Combining this with Eq. (8), and integrating from $T_{\mathrm{H}}, Q=1$, to $T_{\mathrm{C}}, Q_{\max }$, one obtains

$Q_{\max }-1=-\frac{\Delta M a D}{M f D_{\mathrm{eff}}} R^{-1}\left(T_{\mathrm{C}}^{-1}-T_{\mathrm{H}}^{-1}\right) \varphi\left(E_{\mathrm{m}}-E_{\mathrm{v}}\right)$.

In a liquid the defects are "blurred out", so that the BARDEEN-Herring correlation factor $f$ is probably very near to unity (as a lowest limit one can use the solid-state approximation ${ }^{3} f=1-2 / Z$, where $Z$ is the coordination number, about 10 in liquids, making $f=0.8$ ). It can also be presumed that $D^{\prime}$ is small, as special precautions were taken ${ }^{2}$ to limit convection. Assuming therefore $f \cong 1$, $D / D_{\text {eff }} \cong 1$ and, as motivated above, $a \cong-0.1$, one can obtain the "heat of transport" from Eq. (12), expressed as

$$
\varphi\left(E_{\mathrm{m}}-E_{\mathrm{v}}\right)=-20(M / \Delta M) \frac{Q_{\max }-1}{T_{\mathrm{c}}^{-1}-T_{\mathrm{H}}^{-1}}-1 .
$$

When the experimental results for lithium and gallium are substituted into RHS of Eq. (13), $\varphi\left(E_{\mathrm{m}}-E_{\mathrm{v}}\right)$ becomes $-13.5 \mathrm{kcal} / \mathrm{mole}$ for $\mathrm{Li}$ and $-11.5 \mathrm{kcal} / \mathrm{mole}$ for $\mathrm{Ga}$. This is to be compared with the self-diffusion activation energies $\left(E_{\mathrm{m}}+E_{\mathrm{v}}\right)$, $2.8 \mathrm{kcal} / \mathrm{mole}^{9}$ for $\mathrm{Li}$ and $1.1 \mathrm{kcal} / \mathrm{mole}^{15}$ for $\mathrm{Ga}$. The negative values of the "heat of transport" indicate that $E_{\mathrm{v}}$ is considerably greater than $E_{\mathrm{m}}$; if it is assumed, for simplicity and in accordance with a very relaxed model, that $E_{\mathrm{m}} \cong 0$, one finds $\varphi_{\mathrm{Li}}=4.8$ and $\varphi_{\mathrm{Ga}}=10.5$. If $E_{\mathrm{m}} \neq 0$, these values naturally become even greater. The mean displacement length is thus considerably smaller than the distance between the centre of the diffusing species and that of the void. The latter can consequently be thought of as situated at the "outskirt" of the cluster, and the mean displacement of the cluster turns out to be in $\mathrm{Li}$ about $10.4 \%$, in $\mathrm{Ga}$ about $4.8 \%$ of its "diameter".

Thus as the result of this treatment of thermal diffusion data of pure liquid metals, a transport mechanism is suggested, where particles ("clusters") composed of several atoms move into relatively small voids, without any or with a very small activation energy of motion.

\section{d) Free Volume and Cluster Size}

According to a model based on X-ray evidence ${ }^{4}$, a simple liquid (at its m.p.) can be considered as a quasi-b.c.c. array of atoms, with a little less than $10 \%$ of the volume consisting of statistically dispersed voids, or "free volume". Other authors ${ }^{8}$ have also stressed the importance of free volume as the typical feature of fluidity.

One can hence imagine the liquid as a mixture of close-packed clusters, loosely bound single atoms and multishaped voids. Normally the voids are not localized at lattice-places. However, a statistical density fluctuation, squeezing an amount of atoms into a close-packed cluster, may simultaneously create the possibility for the cluster to move, by localizing the corresponding free volume. If a mean size void is created by the close-packing of $n$ atoms, its volume will be

$$
n V_{\mathrm{f}} / N=A \delta,
$$

14 cf. K. Wirtz, Z. Naturforschg. 3 a, 672 [1948].

15 J. Petit and N. H. Nachtrieb, J. Chem. Phys. 24, 1027 [1956]. 
where $V_{\mathrm{f}}$ is the molar free volume, $N$ Avogadro's number, $A$ the void cross-section, $\delta$ its depth. The cluster with the volume

$$
n\left(V_{\mathrm{m}}-V_{\mathrm{f}}\right) / N=A \varphi \bar{l}
$$

(where $V_{\mathrm{m}}$ is the mole volume) will be able to move freely a distance $\bar{l} \cong \delta$ into the void, so that

$$
\varphi \cong\left(V_{\mathrm{m}}-V_{\mathrm{f}}\right) / V_{\mathrm{f}} \text {. }
$$

This is in qualitative agreement with free volume data from X-ray evidence quoted above, and also with "activation volume" values as obtained ${ }^{16}$ by diffusion studies of liquids under pressure.

It is reasonable ${ }^{8}$ to assume, that the opening up of an atomic size void will enable a neighbor atom to drift into it. The amount of atoms which must cooperate to liberate such a suitable void volume is then

$$
n=N \cdot \pi d^{3} / 6 V_{\mathrm{f}}=\left(V_{\mathrm{m}}-V_{\mathrm{f}}\right) / V_{\mathrm{f}},
$$

or

$$
n \cong \varphi \text {. }
$$

Admittedly such an estimate assumes the cluster to be composed of cooperating atoms moving through distances ranging from $+d$ down to comparable ne-

16 N. H. Nachtrieb, in Liquid Metals and Solidification, Amer. Soc. Metals 1958, p. 49. gative values. It has not, however, been postulated in the preceding treatment, that $l$ is necessarily the displacement of all the atoms of the cluster; $\vec{l}$ can equally well be conceived as a mean value, and the cluster as a kind of moving micro-whirl.

Assuming accordingly about 10 atoms to cooperate along the diffusion path, one finds that $\varphi \bar{l}$ is between 2.2 and 3 atomic diameters, depending on whether the cluster is spheric or prolate. Thermal diffusion experiments then indicate that the mean diffusive displacement is between some $10 \%$ and $30 \%$ of an atomic diameter.

It has been noted above, that the present results are compatible with evidence obtained by other experimental means ${ }^{4,10,16}$. It should be mentioned that the existence of clusters is also suggested by a model based on neutron scattering data ${ }^{17}$. According to this model, the cluster size should decrease slowly with rising temperature. This can be seen to agree with Eq. (17) above; $n$ goes down as $V_{\mathrm{f}}$ increases with thermal expansion.

I am indebted to Prof. H. B. Huntington, Troy, N.Y., and to Prof. A. KLemm, Mainz, for valuable discussions.

17 P. A. Egelstaff, Advan. Phys. 11, 203 [1962]. 